Journal

\title{
Terrain classification using intelligent tire
}

\author{
Seyedmeysam Khaleghian ${ }^{\mathrm{a}, *}$, Saied Taheri ${ }^{\mathrm{b}}$ \\ ${ }^{a}$ Department of Biomedical Engineering and Mechanics, Virginia Tech, Blacksburg, VA 24061, United States \\ ${ }^{\mathrm{b}}$ Center for Tire Research (CenTiRe), Virginia Tech, Blacksburg, VA 24061, United States
}

Received 28 August 2016; received in revised form 13 January 2017; accepted 24 January 2017

\begin{abstract}
A wheeled ground robot was designed and built for better understanding of the challenges involved in utilization of accelerometerbased intelligent tires for mobility improvements. Since robot traction forces depend on the surface type and the friction associated with the tire-road interaction, the measured acceleration signals were used for terrain classification and surface characterization. To accomplish this, the robot was instrumented with appropriate sensors (a tri-axial accelerometer attached to the tire innerliner, a single axis accelerometer attached to the robot chassis and wheel speed sensors) and a data acquisition system. Wheel slip was measured accurately using encoders attached to driven and non-driven wheels. A fuzzy logic algorithm was developed and used for terrain classification. This algorithm uses the power of the acceleration signal and wheel slip ratio as inputs and classifies all different surfaces into four main categories; asphalt, concrete, grass, and sand. The performance of the algorithm was evaluated using experimental data and good agreements were observed between the surface types and estimated ones.
\end{abstract}

(C) 2017 ISTVS. Published by Elsevier Ltd. All rights reserved.

Keywords: Wheeled ground robot; Intelligent tire; Terrain classification; Fuzzy logic algorithm

\section{Introduction}

Terrain classification and characterization are the most challenging issues associated with vehicle mobility and more specifically with the mobility of small ground robots. Once the terrain is identified, the robot can adapt to this new surface condition through the onboard traction control system.

Terrain analysis can be classified into two major categories, first terrain classification, aims at associating terrain with well-defined categories, such as asphalt, concrete, gravel and sand. Second, terrain characterization which is determination of the terrain characteristics such as roughness, friction potential, etc. (Ojeda et al., 2006).

Different types of sensors are used in terrain classification. Based on the sensor types, all of the studies in this

\footnotetext{
* Corresponding author.

E-mail address: meysam@vt.edu (S. Khaleghian).
}

field are divided into two main categories; the uses of contact sensors and non-contact sensors. In the studies with the contact sensors, characteristics like signal vibration frequency are used to classify the surface. Park et al. (2012) designed a mobile robot and using the data from the tire sensors. They developed a method to extract the terrain features. Brooks et al. (2005) used a robot with an accelerometer on its chassis. They used the vibration measurement of the chassis to classify the surfaces. Weiss et al. (2006) attached an accelerometer to the vehicle body to measure the vibrations in the direction perpendicular to the ground. Then, they used fast furrier transform (FFT) and power spectral density (PSD) to train a Support Vector Machine (SVM) algorithm to classify the surfaces. They also compared different approaches in vibration based terrain classification (Weiss et al., 2007). DuPont et al. (2005, $2008 \mathrm{~b}, 2008 \mathrm{a})$ attached an accelerometer to the body of an unmanned ground vehicle (UGV). Using the dominant vibration frequency of the UGV's body, they trained a 


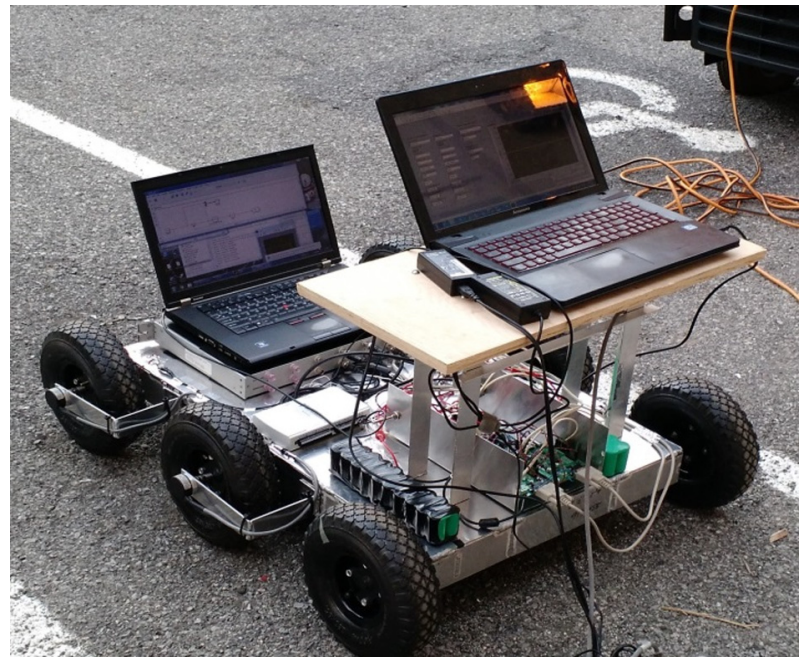

Fig. 1. The six-wheel robot.

Neural Network (NN) to identify the terrain. Collins et al. (2008) presented a vibration-based terrain classification algorithm for a UGV by mapping the vibration outputs to the terrain inputs using the Autonomous Guided Vehicle (AGV) vibration transfer function.

Non-contact sensors studies mostly used optical, sonar or acoustic sensors for terrain classification/characteriza tion purpose. Manduchi et al. (2005) used two sensor systems; a color stereo camera and a single axis Laser Detection and ranging (LADAR) that complement each other. Using stereo range measurements, they developed a color-based classification system to classify the detected terrains (Bellutta et al., 2000; Castano et al., 2001; Talukder et al., 2002). Larson et al. (2004) used a single camera and developed a new terrain classification technique. Vandapel et al. (2004) used a rover with a 3-D LADAR for terrain classification. Lee et al. (2011) used a Charge Coupled Device (CCD) camera; they extracted colors and textures from sensor data and classified the surfaces. Lu et al. (2011) used a laser stripe-based structured light sensor, which already has infrared camera component that allows sensing at night without external lightening, to sense the terrain directly. Their classification does not rely on color measurement, which can be distorted due to weather conditions and illumination. Their method was based on spatial frequency from range data and texture from camera data. Many studies have used a combination of contact and non-contact sensors for terrain classification. Ojeda et al. (2006) used a small robot for terrain classification purposes. Using a gyro with build in accelerometer attached to the robot's chassis, wheel encoder, microphone, infrared sensor and ultra-sonic range sensor, they developed an algorithm to classify different terrain to commonly known classes like gravel, sand and asphalt.

Also, much research has been conducted to characterize different terrains (Bekker, 1956, 1960; Bekker et al., 1969). Howard and Seraji (2001) used a mobile robot with a (a)

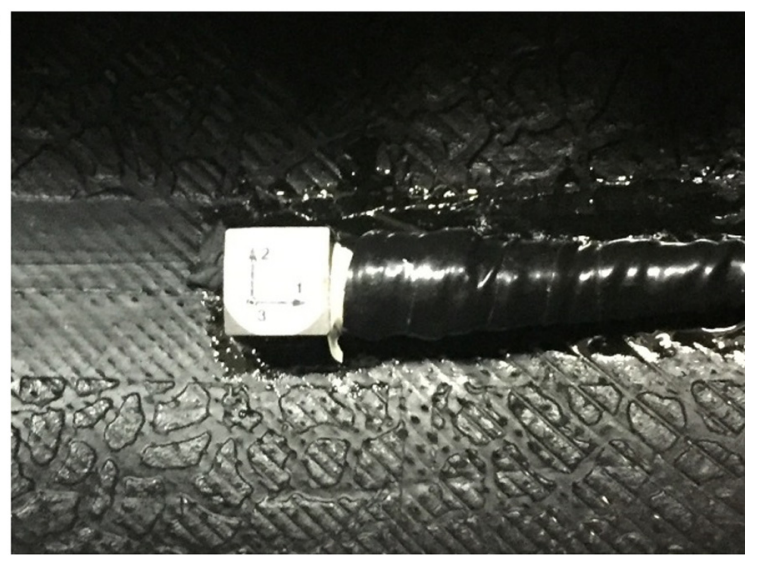

(b)

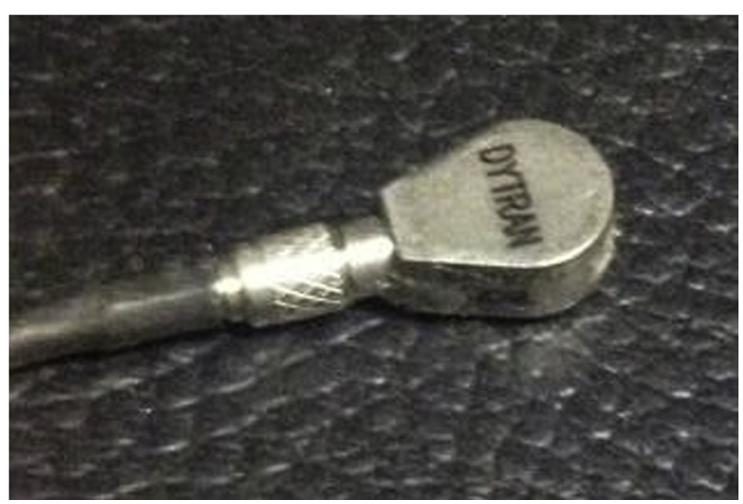

Fig. 2. Acceleration sensors used in the robot: (a) tri-axial accelerometer attached to the tire inner liner, (b) single axis accelerometer attached to the robot's chassis.

vision system and used an Artificial Neural Network (ANN) for real time terrain characterization. Cuong et al. (2014) designed a test rig and used it to measure the vertical damping ratio of tire-soil system using freevibration logarithmic decay method.

In this study, a six-wheel small ground robot was designed and built to classify different surfaces. The robot was equipped with intelligent tire to monitor the interaction between the tire and different terrains. Using accurate encoders, attached to the driven and non-driven wheels of the robot, wheel slip was calculated accurately. Analyzing the sensors data for different speeds on different surfaces, a Fuzzy Logic algorithm was developed to classify different terrains into four main categories; asphalt, sand, concrete, and grass. The rest of this paper is structured as follows. Different parts of the robot and its data collecting system and speed controller algorithm are introduced in Section 2. The fuzzy logic based terrain classification algorithm is explained -in Section 3. Results and discussion is presented in Section 4 follows by conclusions in Section 5 .

\section{Robot design}

An all-terrain mobile robot platform (ATR) was designed and built in this study. The chassis base is $14.25^{\prime \prime}$ wide $\times 17.25^{\prime \prime}$ long, which are surrounded by $2.13^{\prime \prime}$ 


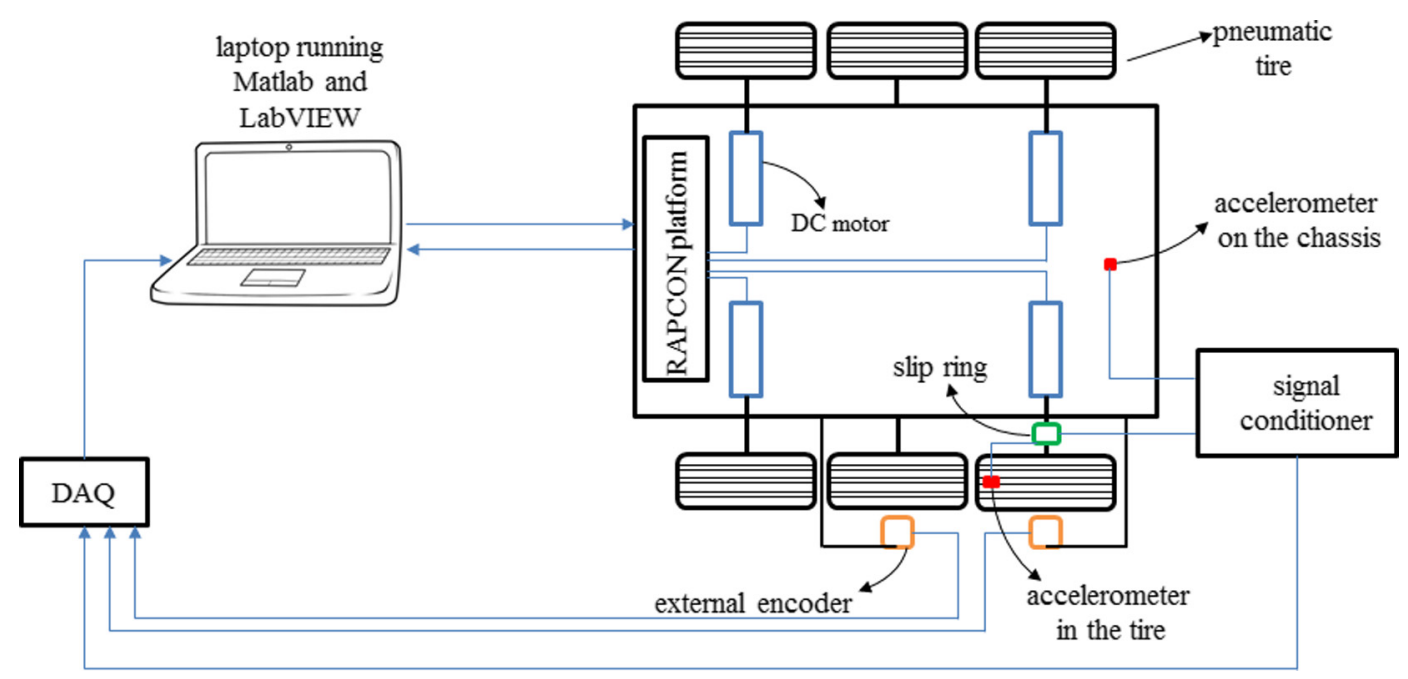

Fig. 3. Schematic of the robot and data collecting system.

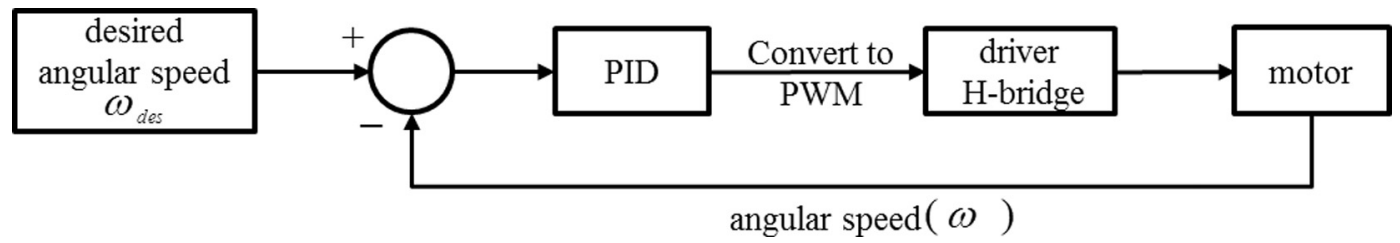

Fig. 4. The robot's speed control algorithm.

Table 1

Specification of the fuzzy algorithm, used in this study.

\begin{tabular}{ll}
\hline Definition of AND & $\operatorname{Min}, \mu_{A \cap B}(x)=\min \left(\mu_{A}(x), \mu_{B}(x)\right)$ \\
Definition of OR & $\operatorname{Max}, \mu_{A \cup B}(x)=\max \left(\mu_{A}(x), \mu_{B}(x)\right)$ \\
Implication method & Mamdani, truth value $=\min \left(f_{a}(x), f_{b}(x)\right)$ \\
Defuzzification method & Centroid, Decision $=\frac{\int_{s} y \mu(u) d y}{\int_{s} \mu(u) d y}$, which $\mu(u)$ is the result of the rules were added together \\
\hline
\end{tabular}

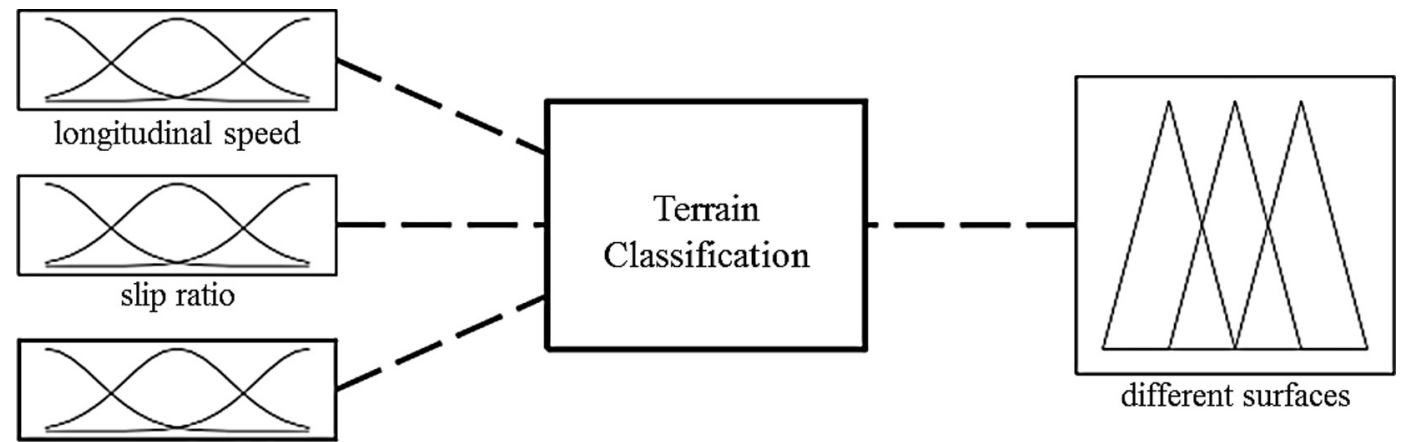

energy of radial acceleration signal

Fig. 5. The terrain classification fuzzy diagram.

height sidewalls. The chassis provides the options for 4WD and 6WD robot platform and provides enough space for the wheel shafts to be coupled directly to the motors. Four $24 \mathrm{~V}$ brushed permanent magnet DC motors with planetary gearbox from Shayang Ye Industrial Company (sup- plied by supper droid robots) were used. Based on the information that was provided by Supper Droid, the rated speed for the motors is $285 \mathrm{RPM}$, the rated torque is $11 \mathrm{kgf}-\mathrm{cm}$ and the reduction ratio is 1:12. Each motor has a dual channel/quadrature encoder with 900 pulses 


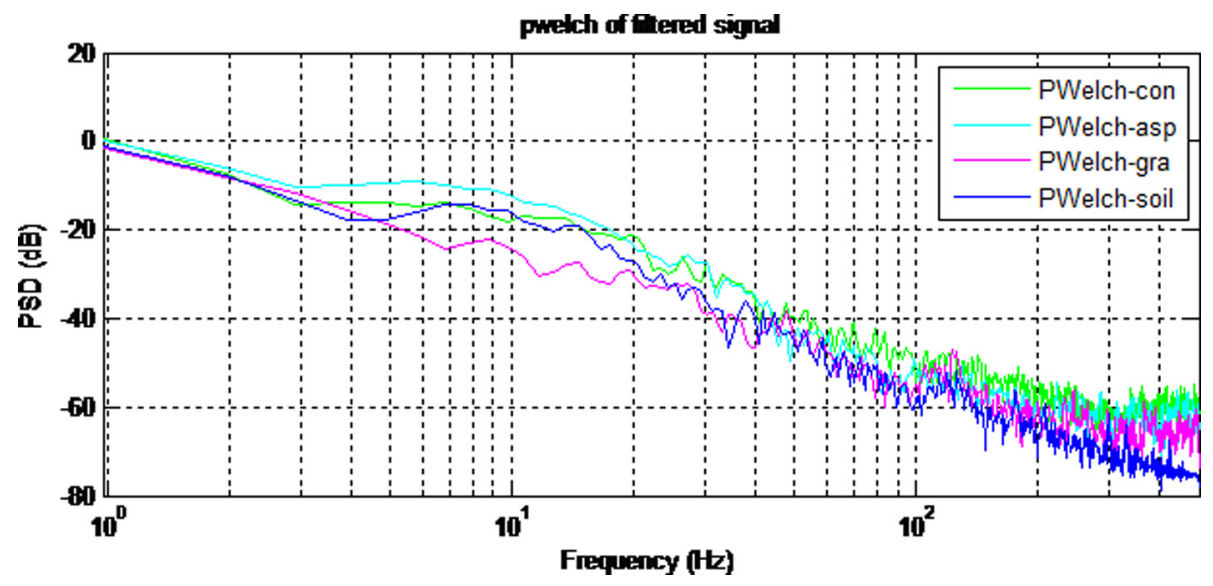

Fig. 6. Power spectral density of the acceleration data on different surfaces.

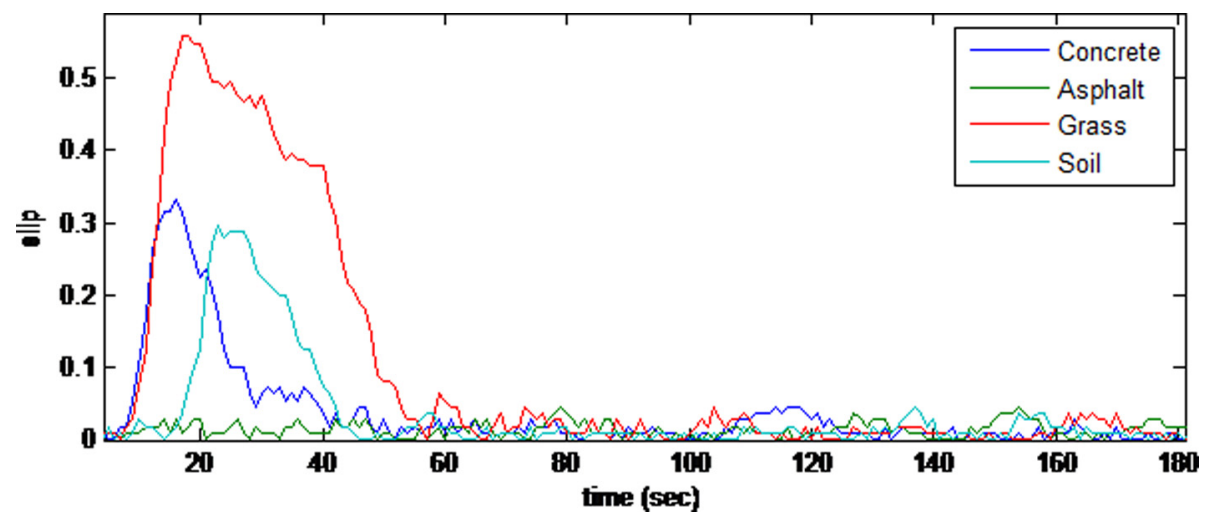

Fig. 7. Slip-time, calculated from the speed of driven and non-driven wheels on different surfaces.

Table 2

Design of experiment for surface classification study.

\begin{tabular}{ll}
\hline Surface type & Wheel speed $(\mathrm{rpm})$ \\
\hline Asphalt & $30,50,70,90$ \\
Concrete & $30,50,70,90$ \\
Grass & $30,50,70,90$ \\
Soil & $30,50,70,90$ \\
\hline
\end{tabular}

per revolution, mounted to the tail shaft of the motor for speed control purposes (Shayang et al., 2000). The completed robot is shown in Fig. 1.

Two RAPCON platforms form ZELTOM, which offer a seamless interface between physical plants and Matlab/ Simulink for implementation of different control algorithms in real-time, were used to control the motors. Each of RAPCON platforms has two channels to communicate and control two DC motors simultaneously and provides the means for real time operation with sampling rate up

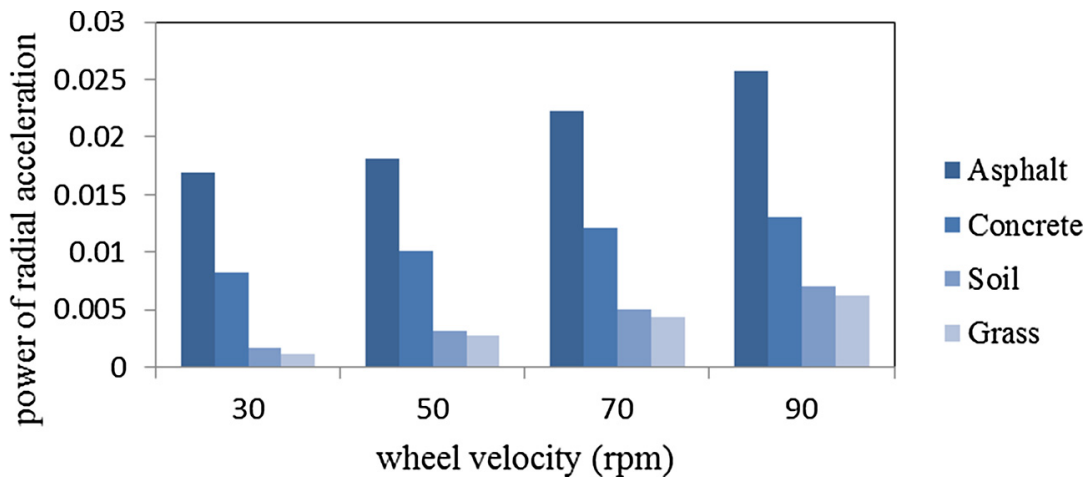

Fig. 8. Power of acceleration signal for different wheel speed on different surfaces. 


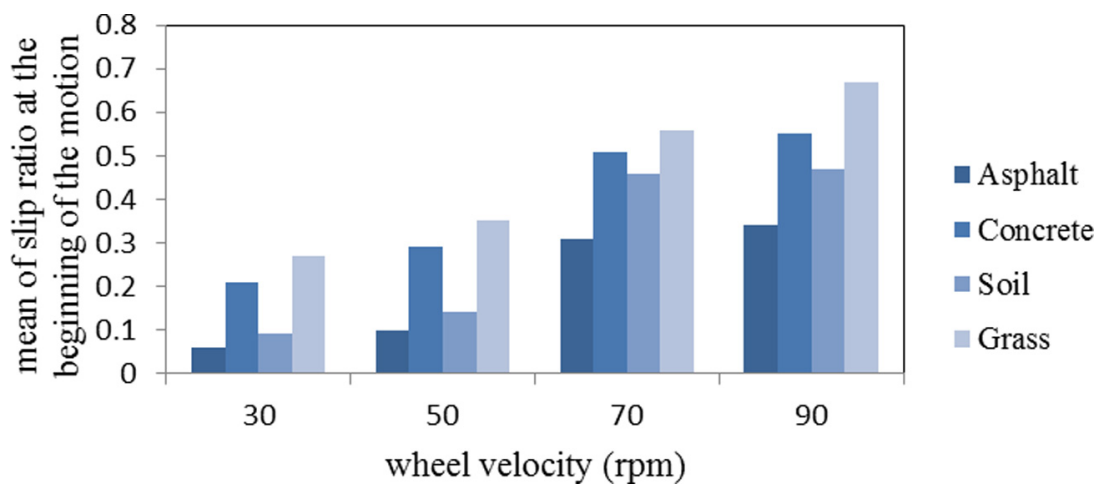

Fig. 9. Mean of slip ratio for different wheel velocities on different surfaces.

to $15.2 \mathrm{kHz}$. In order to control the motion of each wheel separately, two RAPCON Platforms were used at the same time. Also two 20 cell ( $24 \mathrm{~V}$ - DC, $4500 \mathrm{~mA} \mathrm{~h}$ ) rechargeable NiHM battery packs were used for each of the motor controllers. This provides the required energy for the robot to operate for a minimum of one hour at the highest speed on a concrete road surface.

Six pneumatic tires were used under the robot; four of them are directly attached to the motors and the middle ones roll freely. The tires are $10 \mathrm{in}$. in diameter and $4 \mathrm{in}$. wide, which are rated at $30 \mathrm{psi}$ with the maximum load capacity of $300 \mathrm{lb}$. Since the robot has sensors inside the right front and left rear tires, slip ring was used to transmit the electrical signal from the rotating wheel to stationary data acquisition system and vice versa. A SRA-73683 slip ring with 6 input-output circuits from Moog was used. The inner bore for this slip ring was $0.5^{\prime \prime}$, made it possible to be mounted on the wheel shaft. The maximum speed for this slip ring is 120 RPM and the maximum voltage is $120 \mathrm{~V}$ (Moog Components Group, 2012).As was mentioned before, the robot was designed for terrain classifica tion/identification. For this purpose, the robot was instrumented with many sensors which are explained briefly next. The intelligent tire technology was developed at the Center for Tire Research (CenTiRe) and was utilized for this project (Singh et al., 2013, 2012; Khaleghian et al., 2016). A 3023A5 Tri-axial accelerometer from Dytran was fixed inside the robot's tire using a rubber patch, shown in Fig. 2a, to monitor the vibration of the contact patch in three directions. The sensitivity of the tri-axial accelerometer is $10 \mathrm{mv} / \mathrm{g}$ and its measuring range is $\pm 500 \mathrm{~g}$.

A Dytran 3225F1 single axis accelerometer, with the sensitivity of $10 \mathrm{mv} / \mathrm{g}$ and the measuring range of $\pm 500 \mathrm{~g}$, was attached to the chassis in order to monitor the dynamics of the robot in vertical direction. The single axes accelerometer is shown in Fig. 2b. To power the accelerometer, Dytran Model 4116 signal conditioner/amplifier was used.

To measure the angular speed and the slip of the wheel accurately, two model $15 \mathrm{~S}$ encoders, with the accuracy of 10,000 pulses per revolution, from Encoder Product Company were attached to a driven and un-driven wheel of the robot externally. The slip ratio was calculated as follows (Gustafsson, 1997):

$$
\begin{array}{ll}
s=\frac{\omega_{w} r_{w}-v_{w}}{v_{w}} & \text { during braking } \\
s=\frac{\omega_{w} r_{w}-v_{w}}{\omega_{w} T_{w}} & \text { during accelerating }
\end{array}
$$

where $\omega_{w}$ is the angular speed of driven wheel, $r_{w}$ is the effective radius of the wheel and $v_{w}$ is the longitudinal speed of the robot, calculated as $\omega r_{w}$, where $\omega$ is the angular speed of un-driven wheel.

\subsection{Data acquisition system}

NI USB 6218 data acquisition system was used to collect data from all of the sensors. This is a 16-bit, $250 \mathrm{kS} / \mathrm{s} \mathrm{USB}$ devices with 32 analog inputs and 8 digital inputs and two 32-bit counters. The accelerometers were connected to the analog input channels and the encoders were attached to the counter/timer pins of this device. It measures a number of time-related quantities, counting events or totalizing, and monitoring position with quadrature encoders. A data collecting routine was developed using LabVIEW to collect the time synchronized data of all sensors with the same sample rate, $1000 \mathrm{~Hz}$, for this study. Fig. 3 shows the schematic of the robot and data collecting system.

\subsection{Speed control algorithm}

RAPCON platform was used to control the motors, which works perfectly with Matlab/Simulink real-time toolbox. A simple PID controller was designed in Matlab/Simulink, shown in Fig. 4, to control the speed of each wheel separately, which uses the encoder signal of the motors as the feedback. The PID parameters were tuned by trial and error to achieve the best performance in both high and low speed.

\section{Methodology}

In this study a Fuzzy logic algorithm was developed and used to classify different terrains. Fuzzy logic is an extension of Boolean logic by Zadeh (1999) and Hájek (1998) which provides a very valuable flexibility for reasoning. 
(a)
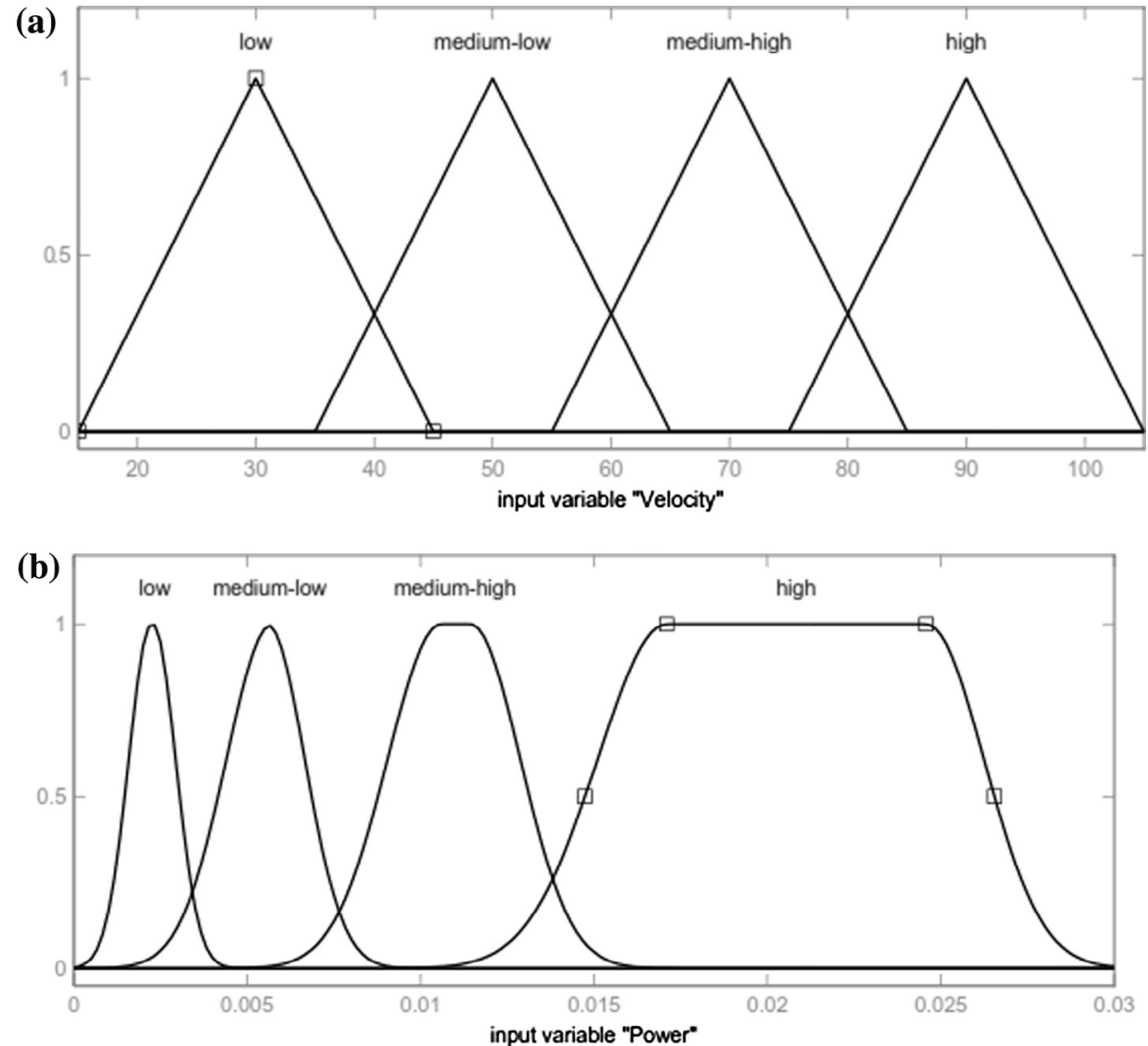

(c)

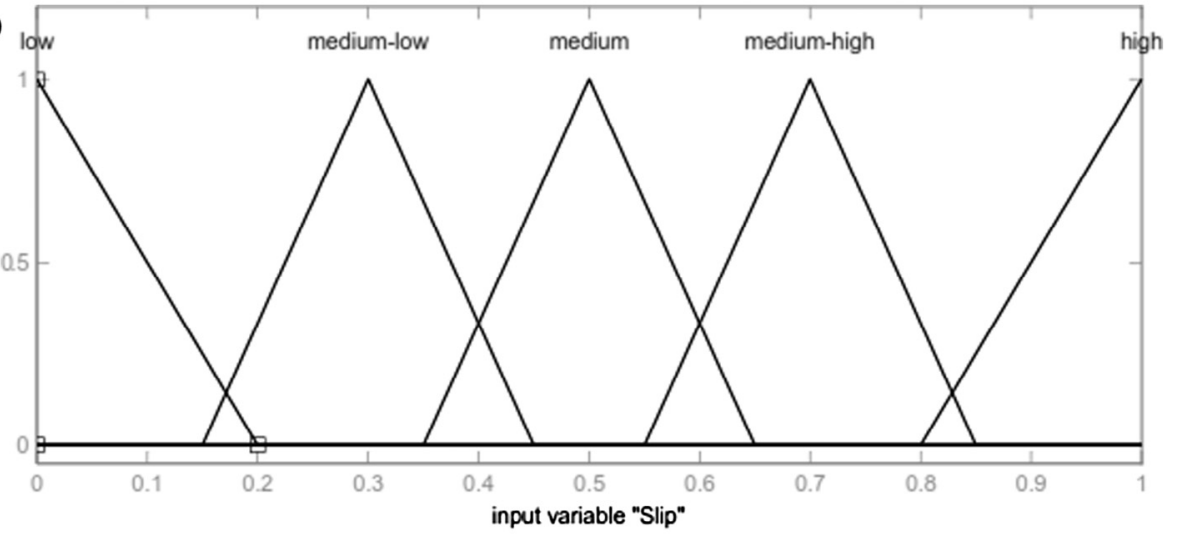

(d)

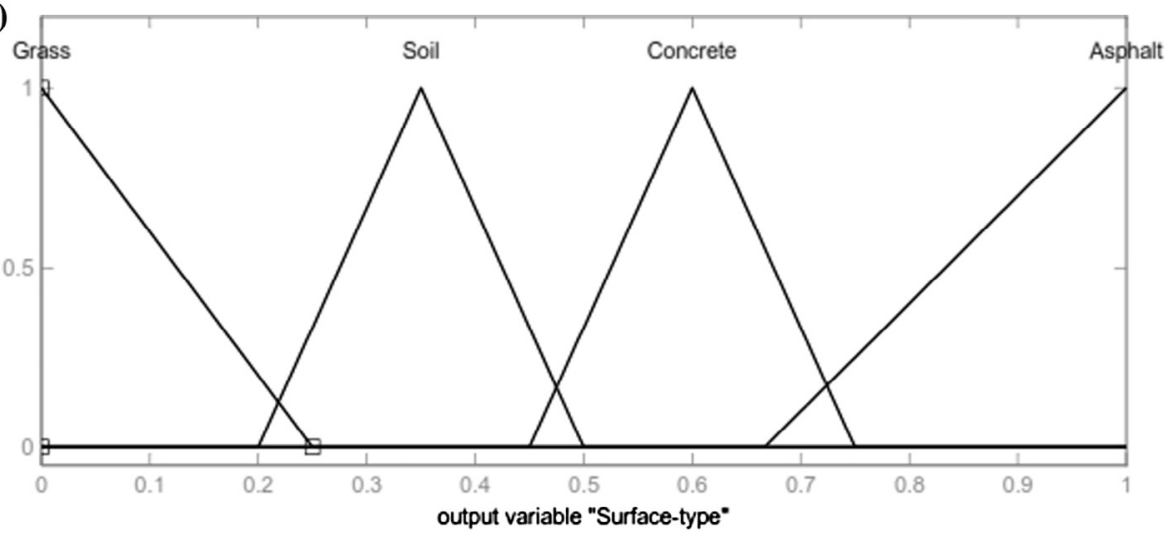

Fig. 10. Fuzzy set selected for: (a) input variable "wheel velocity", (b) input variable "power of acceleration", (c) input variable "wheel slip" and (d) output variable "surface type". 

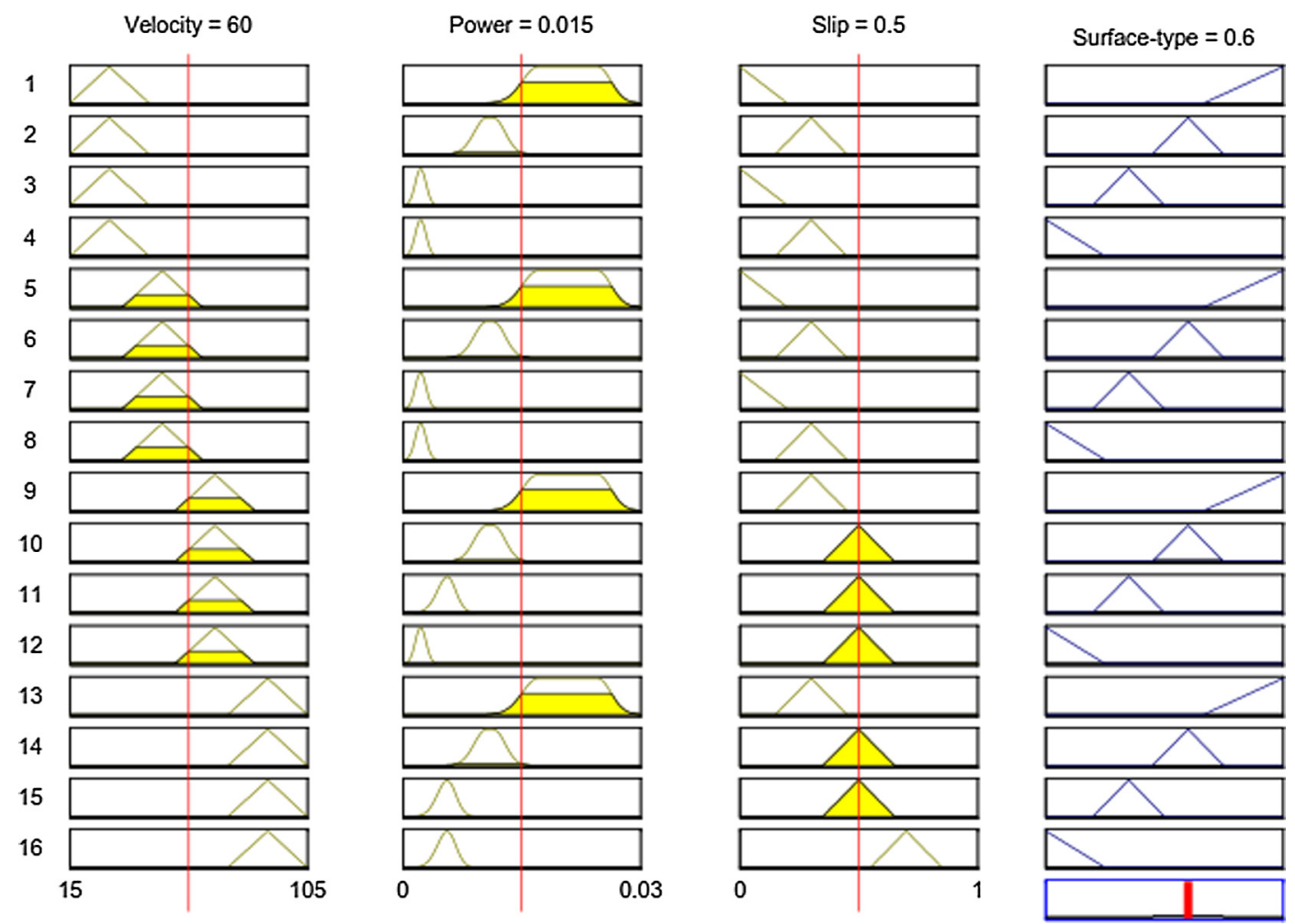

Fig. 11. Fuzzy rules defined for terrain classification purpose base on collected data.

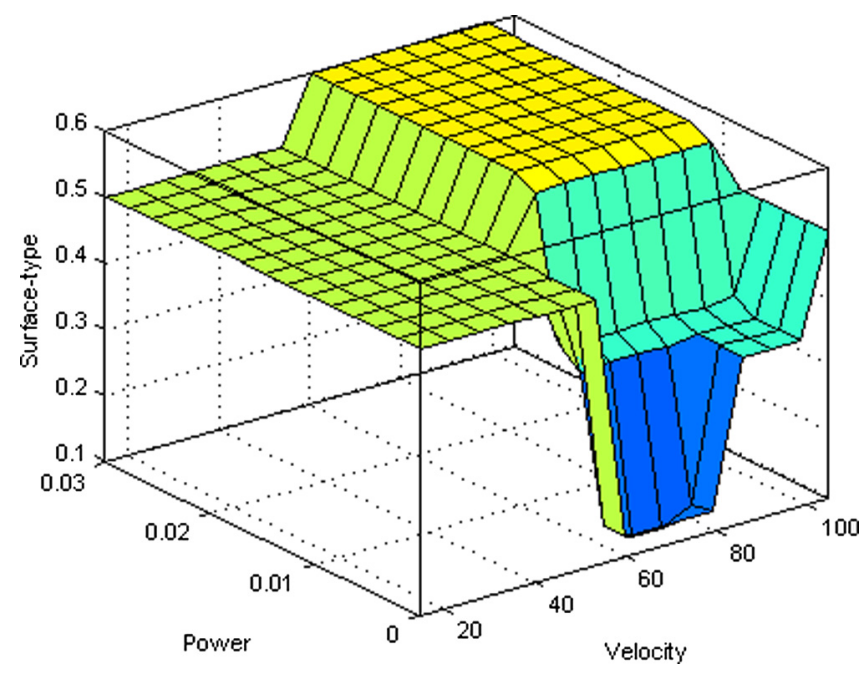

Fig. 12. The surface viewer of terrain classification algorithm.

Table 3

Design of experiment for surface classification study.

\begin{tabular}{ll}
\hline Surface type & Wheel speed $(\mathrm{rpm})$ \\
\hline Asphalt & $40,60,80$ \\
Concrete & $40,60,80$ \\
Grass & $40,60,80$ \\
Soil & $40,60,80$ \\
\hline
\end{tabular}

The shape of membership functions was chosen arbitrary using the result of statistical analysis or by following the advice of the expert and can be assigned as sigmoid, Gaussian, hyperbolic, exponential, tangent or any other desired forms.

The definitions of operators on fuzzy sets, the method of implication, and the defuzzification method are not always the same in Fuzzy logic systems and can be chosen based on the problem. The specification of the Fuzzy Logic algorithm used for this study is shown in Table 1 , in which the membership function of a fuzzy set $A$ is shown as $\mu_{A}(x)$.

The Fuzzy logic system used for this study is shown in Fig. 5. Using the robot's wheel velocity, mean of wheel slip ratio at the beginning of the motion and energy (power) of radial acceleration (from the accelerometer embedded to the tire inner liner) as inputs; the surfaces are classified into four categories: Grass, sand, asphalt and concrete.

The energy (power) of a signal $x(t)$ is calculated as follows:

$$
\begin{aligned}
& E_{s c}=\langle x(t), x(t)\rangle=\int_{-\infty}^{\infty}|x(t)|^{2} d t \\
& E_{s d}=\langle x(t), x(t)\rangle=\sum_{-\infty}^{\infty}|x(t)|^{2} d t
\end{aligned}
$$

where $E_{s c}, E_{s d}$ are the energy of continuous-time and discrete- time signal $x(t)$ respectively. The wheel slip ratio is calculated using Eq. (1).

The six-wheeled ground robot was used to develop a fuzzy logic based surface classification algorithm. It was 


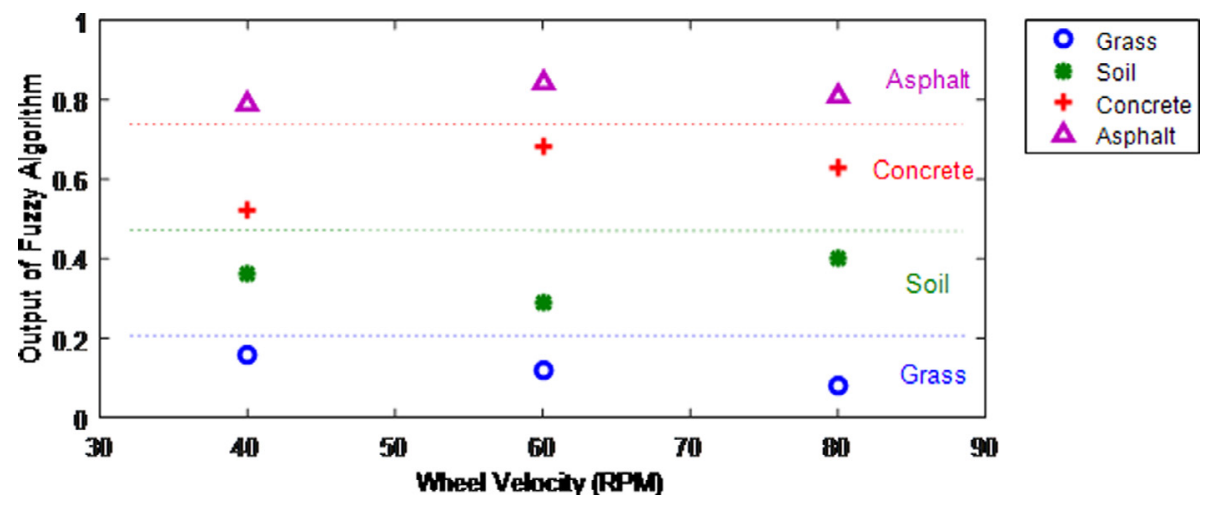

Fig. 13. The output of terrain classification algorithm for validation set.

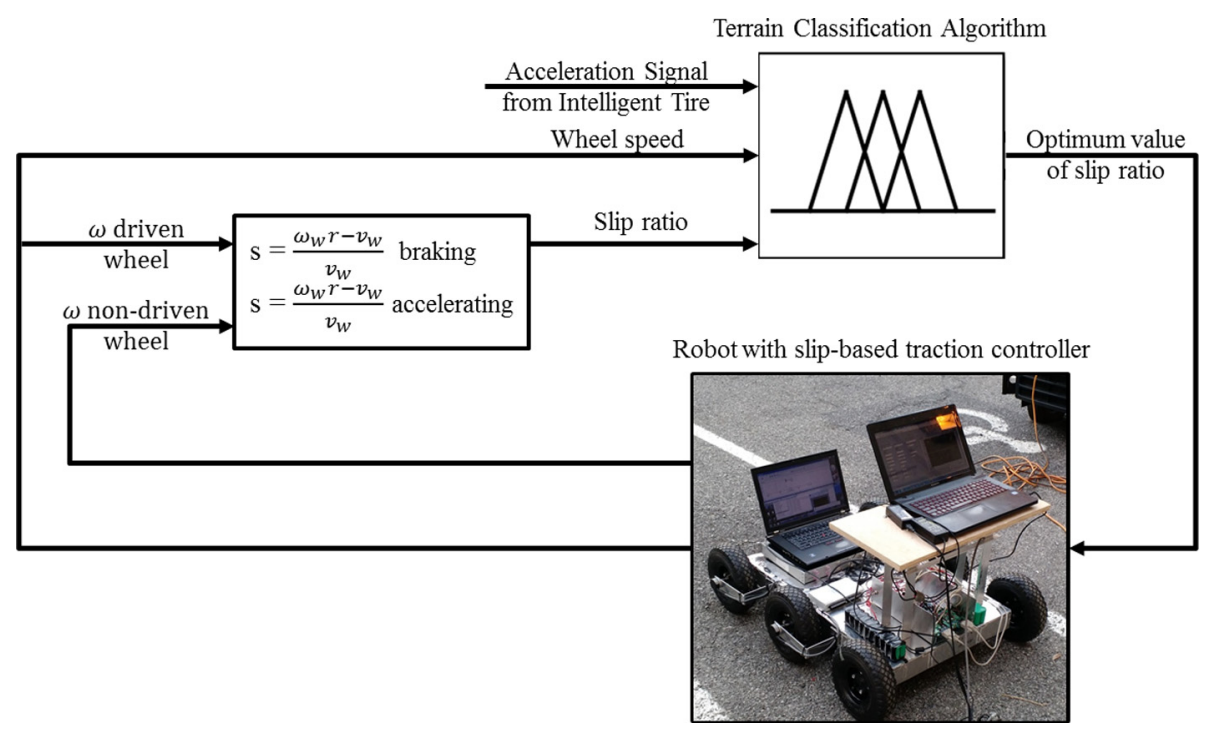

Fig. 14. Sample intelligent tire based traction controller algorithm.

observed that the power of the acceleration signal, acquired from the interaction between the robot's tire and different surfaces is different (mainly) for the frequencies below $20 \mathrm{~Hz}$. Fig. 6 shows the power spectral density of the acceleration signal for the same sample commanded speed on different surfaces (calculated using pwelch command in Matlab).

Also, the slip ratio at the beginning of the motion, calculated from the velocity of driven and non-driven wheels in specific commanded velocity, is the other parameter which changes on different surfaces and is used to classify different surfaces. Fig. 7 depicts wheel slip ratio on different surfaces for the same sample commanded speed. As it is observed, these two parameters give a powerful tool to distinguish between different surfaces in specific commanded speeds.

\section{Results and discussion}

To classify different surfaces, an experiment was designed with four different longitudinal speeds on four dif- ferent surfaces, concrete, asphalt, grass and soil. Results were used to define the fuzzy rules. Table 2 shows the design of experiment for this study.

Power of acceleration signal and the mean values of slip ratio at the beginning of motion for different robot wheel speed are shown in Figs. 8 and 9 respectively.

These two parameters, power of radial component of acceleration signal (below $20 \mathrm{~Hz}$ ) and the mean of the slip ratio value at the beginning of the motion, along with robot's wheel velocity are considered as the inputs of the fuzzy algorithm and the surface type is the output, Mamdani was used as the implication method.

The fuzzification was done using the collected data; Fig. 10 shows the fuzzy sets selected for the inputs and the output of this fuzzy system.

Fig. 11 shows the list of fuzzy rules used for this study and the decision making process for a specific case. Fuzzy rules were defined based on the collected data. For instance, for the wheel speed of 30 , the power of the signal on the asphalt was 0.0169 and the maximum slip ratio was 0.06 so the following fuzzy rule was defined: 
If the speed is "low" and the power is "high" and the slip is "low" then the surface is "asphalt"

The surface viewer of terrain classification algorithm is shown in Fig. 12 that shows that power of the radial component of acceleration signal reaches its highest values on asphalt and its lowest values on grass. This was predictable, since the vibration of the tire, caused by the contact between the tire and the road, is damped on surfaces with softer texture. Also, power of radial vibration is higher when the robot's speed is increased.

In order to validate the terrain classification algorithm, another experiment is designed with three different commanded speeds (rather than the ones were used to terrain the algorithm) on each of the surfaces; the data of five tire revolutions was used to calculate the power of acceleration signal. Table 3 shows design of experiment to validate the algorithm.

The output of proposed fuzzy logic based terrain classification algorithm for the validation set is shown in Fig. 13, the borders of surface types are based on the membership function that was presented in Fig. 10d. The wheel velocity, the power of radial acceleration signal (below $20 \mathrm{~Hz}$ ) for five tire revolutions and the mean value of slip ratio at the beginning of the motion were used as the inputs. As it is observed, the algorithm successfully identified the surfaces for different velocities.

Once the surface was identified based on the optimum value of slip ratio on each surface, a desired traction controller can be designed for the robot, a sample algorithm for this purpose is shown in Fig. 14.

\section{Conclusion}

Identification of the surface that the UGV (unmanned ground vehicle) is running on is the key to design a more efficient controller for this type of vehicle. Once the surface is identified, the appropriate traction and stability control strategies can be selected which leads to improved mobility of UGVs. Intelligent tire, a tri axial accelerometer embedded to the tire innerliner, can be used as a tool to identify different surfaces based on monitoring the interaction between the tire and the road.

To study the application of intelligent tire on the mobility of UGV's, a six-wheel ground robot was designed and built. The robot was instrumented with appropriate sensors; two high resolution encoders $(10,000$ pulses per revolution) are attached to one of the driven and a non-driven wheel of the robot in order to measure the wheel slip ratio. Also, there is a tri-axial accelerometer inside the tire to capture the tire vibration caused by the tire-road contact.

Testing the robot on different surfaces, it was observed that the power of the radial component of acceleration signal, from the accelerometer inside the tire is different on different surfaces (especially for the frequencies below $20 \mathrm{~Hz}$ ). Also, the values of slip ratio at the beginning of motion (with same final speed) were different; the higher the friction of the surface, the lower the value of slip ratio.
A fuzzy logic based terrain classification algorithm was developed, using the power of the radial component of acceleration signal from intelligent tire, the mean value of slip at the beginning of the motion and wheel speed as the inputs, the surfaces were classified into four different categories: asphalt, concrete, soil and grass.

To develop the algorithm an experiment was designed with four different speeds and the fuzzy rules was extracted based on the test results. To test the accuracy of the algorithm another set of data with three different speeds, rather than the ones were used to develop the algorithm, was used. In all the cases the proposed algorithm identified the surface correctly and the algorithm was validated.

\section{References}

Bekker, M., 1956. Theory of Land Locomotion: The Mechanics of Vehicle Mobility. University of Michigan Press, Ann Arbor, MI.

Bekker, G., 1960. Off-road Locomotion. University of Michigan Press, Ann Arbor, MI.

Bekker, M.G., 1969, Introduction to terrain-vehicle systems.

Bellutta, P., Manduchi, R., Matthies, L., Owens, K., Rankin, A., 2000. Terrain perception for DEMO III. In: Proc. Intelligent Vehicles Symposium, 2000. IV 2000. Proceedings of the IEEE. IEEE, pp. 326331.

Brooks, C., Iagnemma, K., Dubowsky, S., 2005. Vibration-based terrain analysis for mobile robots. In: Proc. Proceedings of the 2005 IEEE International Conference on Robotics and Automation. IEEE, pp. $3415-3420$.

Castano, R., Manduchi, R., Fox, J., 2001, Classification experiments on real-world texture.

Collins, E.G., Coyle, E.J., 2008. Vibration-based terrain classification using surface profile input frequency responses. In: Proc. IEEE International Conference on Robotics and Automation, 2008. ICRA 2008. IEEE, pp. 3276-3283.

Cuong, D.M., Zhu, S., Ngoc, N.T., 2014. Study on the variation characteristics of vertical equivalent damping ratio of tire-soil system using semi-empirical model. J. Terramech. 51, 67-80.

DuPont, E.M., Roberts, R.G., Selekwa, M.F., Moore, C.A., Collins, E. G., 2005. Online terrain classification for mobile robots. In: Proc. ASME 2005 International Mechanical Engineering Congress and Exposition. American Society of Mechanical Engineers, pp. 16431648.

DuPont, E.M., Collins, E., Coyle, E.J., Roberts, R.G., 2008, Terrain classification using vibration sensors: theory and methods. New Research on Mobile Robotics.

Dupont, E.M., Moore, C.A., Collins Jr, E.G., Coyle, E., 2008 b. Frequency response method for terrain classification in autonomous ground vehicles. Autonom. Robots 24 (4), 337-347.

Gustafsson, F., 1997. Slip-based tire-road friction estimation. Automatica 33 (6), 1087-1099. Jun 30.

Hájek, P., 1998. Metamathematics of Fuzzy Logic. Springer Science \& Business Media.

Howard, A., Seraji, H., 2001. Vision-based terrain characterization and traversability assessment. J. Robot. Syst. 18 (10), 577-587.

Khaleghian, S., Ghasemalizadeh, O., Taheri, S., 2016. Estimation of the tire contact patch length and normal load using intelligent tires and its application in small ground robot to estimate the tire-road friction. Tire Sci. Technol. 44 (4), 248-261.

Larson, A.C., Voyles, R.M., Demir, G.K., 2004. Terrain classification through weakly-structured vehicle/terrain interaction. In: Proc. 2004 IEEE International Conference on Robotics and Automation. ICRA'04. IEEE, pp. 218-224.

Lee, S.-Y., Kwak, D.-M., 2011. A terrain classification method for UGV autonomous navigation based on SURF. In: Proc. 2011 8th Interna- 
tional Conference on Ubiquitous Robots and Ambient Intelligence (URAI). IEEE, pp. 303-306.

Lu, L., Ordonez, C., Collins, E.G., Coyle, E., Palejiya, D., 2011. Terrain surface classification with a control mode update rule using a 2D laser stripe-based structured light sensor. Robot. Autonom. Syst. 59 (11), 954-965.

Manduchi, R., Castano, A., Talukder, A., Matthies, L., 2005. Obstacle detection and terrain classification for autonomous off-road navigation. Autonom. Robots 18 (1), 81-102.

Moog Components Group, 2012. Slip Rings With Through-Bores. $<$ http://www.moog.com/products/slip-rings/commercial-industrialslip-rings/slip-rings-with-through-bores/sra-73683/>.

Ojeda, L., Borenstein, J., Witus, G., Karlsen, R., 2006. Terrain characterization and classification with a mobile robot. J. Field Robot. 23 (2), 103-122.

Park, B., Kim, J., Lee, J., 2012. Terrain feature extraction and classification for mobile robots utilizing contact sensors on rough terrain. Proc. Eng. 41, 846-853.

Shayang, Y., 2000. Industrial Co., Ltd., Micro DC Planetary Geared Motors.

Singh, K.B., Arat, M.A., Taheri, S., 2012. Enhancement of collision mitigation braking system performance through real-time estimation of tire-road friction coefficient by means of smart tires. SAE Int. J. Passenger Cars-Electron. Electrical Syst. 5 (2), 607-624.

Singh, K.B., Arat, M.A., Taheri, S., 2013. An intelligent tire based tireroad friction estimation technique and adaptive wheel slip controller for antilock brake system. J. Dynam. Syst. Measure. Control 135 (3), 031002.

Talukder, A., Manduchi, R., Castano, R., Owens, K., Matthies, L., Castano, A., Hogg, R., 2002. Autonomous terrain characterisation and modelling for dynamic control of unmanned vehicles. In: Proc. IEEE/RSJ International Conference on Intelligent Robots and Systems, 2002. IEEE, pp. 708-713.

Vandapel, N., Huber, D.F., Kapuria, A., Hebert, M., 2004. Natural terrain classification using 3-d ladar data. In: Proc. 2004 IEEE International Conference on Robotics and Automation. ICRA'04. IEEE, pp. 5117-5122.

Weiss, C., Fechner, N., Stark, M., Zell, A., 2007. Comparison of different approaches to vibration-based terrain classification. In: Proc. EMCR.

Weiss, C., Frohlich, H., Zell, A., 2006. Vibration-based terrain classification using support vector machines. In: Proc. 2006 IEEE/RSJ International Conference on Intelligent Robots and Systems. IEEE, pp. 4429-4434.

Zadeh, L.A., 1999. Fuzzy sets as a basis for a theory of possibility. Fuzzy Sets Syst. 100, 9-34. 